\title{
Implementation of algorithms for tuning parameters in regularized least squares problems in system identification
}

Tianshi Chen and Lennart Ljung

\section{Linköping University Post Print}

\section{Tweet}

N.B.: When citing this work, cite the original article.

Original Publication:

Tianshi Chen and Lennart Ljung, Implementation of algorithms for tuning parameters in regularized least squares problems in system identification, 2013, Automatica, (49), 7, 22132220.

http://dx.doi.org/10.1016/j.automatica.2013.03.030

Copyright: Elsevier http://www.elsevier.com/

Postprint available at: Linköping University Electronic Press http://urn.kb.se/resolve?urn=urn:nbn:se:liu:diva-96405 


\title{
Implementation of Algorithms for Tuning Parameters in Regularized Least Squares Problems in System Identification
}

\author{
Tianshi Chen and Lennart Ljung
}

Division of Automatic Control, Department of Electrical Engineering, Linköping University, Linköping, SE-58183, Sweden

\begin{abstract}
There is recently a trend to study linear system identification with high order finite impulse response (FIR) models using the regularized least-squares approach. One key of this approach is to solve the hyper-parameter estimation problem that is usually non-convex. Our goal here is to investigate implementation of algorithms for solving the hyper-parameter estimation problem that can deal with both large data sets and possibly ill-conditioned computations. In particular, a QR factorization based matrix-inversion-free algorithm is proposed to evaluate the cost function in an efficient and accurate way. It is also shown that the gradient and Hessian of the cost function can be computed based on the same QR factorization. Finally, the proposed algorithm and ideas are verified by Monte-Carlo simulations on a large data-bank of test systems and data sets.
\end{abstract}

Key words: Least squares; Regularization; Empirical Bayes method; Marginal likelihood maximization; QR factorization.

\section{Introduction}

The linear least squares problem to estimate linear regressions is one of the most basic estimation problems, and there is an extensive literature around it, e.g. (Rao, 1973; Daniel \& Wood, 1980; Draper \& Smith, 1981). The problem has a rich structure and new aspects keep being observed and investigated. A recent such trend is to use large linear regressions for estimating impulse responses and models of dynamical systems. This so called system identification problem has in itself an extensive literature mostly based on maximum likelihood techniques and non-convex optimization techniques, e.g. (Ljung, 1999; Söderström \& Stoica, 1989; Ljung, 2010).

It has been observed, (Pillonetto \& Nicolao, 2010; Pillonetto, Chiuso \& Nicolao, 2011; Chen, Ohlsson \& Ljung, 2012; Chen, Zhao \& Ljung, 2012), that it may be beneficial to estimate a high order finite impulse response (FIR) model using regularized least squares, - or, equivalently, Bayesian regression - and perhaps treat this high order FIR model further to find a suitable and practical model. More specifically, consider a single input single output linear stable system

$$
y(t)=G_{0}(q) u(t)+v(t)
$$

where $q$ is the shift operator, $q u(t)=u(t+1), v(t)$ is the

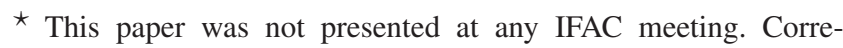
sponding author Tianshi Chen. Tel. +46 (0)13282226. Fax. +46 (0) 13282622 .

Email addresses: tscheneisy. liu. se (Tianshi Chen), l jung@isy. liu.se (Lennart Ljung).
}

noise, independent of the input $u(t)$, and $G_{0}(q)$ is

$$
G_{0}(q)=\sum_{k=1}^{\infty} g_{k}^{0} q^{-k}
$$

The coefficients $g_{k}^{0}, k=1, \ldots, \infty$ form the impulse response of $G_{0}(q)$. Assume we have collected $M$ data points $y(t), u(t), t=1, \cdots, M$. We hope to estimate the impulse response $g_{k}^{0}, k=1, \ldots, \infty$ as well as possible. Since the impulse response decays exponentially for linear stable $G_{0}(q)$, it is often enough to truncate it at certain order and consider

$$
G(q, \theta)=\sum_{k=1}^{n} g_{k} q^{-k}, \quad \theta=\left[\begin{array}{lll}
g_{1} & \cdots & g_{n}
\end{array}\right]
$$

which is the $n$th order FIR model. In (Pillonetto \& Nicolao, 2010; Pillonetto et al., 2011; Chen, Ohlsson \& Ljung, 2012), the parameter vector $\theta$ is estimated in a linear relationship

$$
Y_{N}=\Phi_{N}^{T} \theta+V_{N}
$$

where $N=M-n, \quad Y_{N}, V_{N} \in \mathbb{R}^{N}, \Phi_{N}^{T} \in \mathbb{R}^{N \times n}$ and the $i$ th row of $Y_{N}, V_{N}$ and $\Phi_{N}^{T}$ are $y(n+i), v(n+i)$, and $[u(n+i-1) \cdots u(i)]$, respectively. The estimate of $\theta$ used, $\hat{\theta}_{N}$ is the value that minimizes the regularized least squares criterion

$$
\begin{aligned}
\hat{\theta}_{N} & =\underset{\theta}{\arg \min }\left\|Y_{N}-\Phi_{N}^{T} \theta\right\|^{2}+\sigma^{2} \theta^{T} P(\alpha)^{-1} \theta \\
& =\left(\Phi_{N} \Phi_{N}^{T}+\sigma^{2} P(\alpha)^{-1}\right)^{-1} \Phi_{N} Y_{N}
\end{aligned}
$$

where $\|\cdot\|$ denotes the Euclidean norm, $P(\alpha)$ is the $n \times n$ regularization matrix and the parameter vector $\alpha$ is a $m$ - 
dimensional vector of tuning parameters for the regularization. The meaning of the scalar parameter $\sigma^{2}$ will become clear shortly. In practice, $\alpha$ is not known and has to be estimated from the known $Y_{N}, \Phi_{N}$. There are several ways to estimate $\alpha$, but we shall consider the dominating approach:

$$
\begin{aligned}
\hat{\alpha} \triangleq \underset{\alpha}{\arg \max } p\left(Y_{N} \mid \alpha\right) & \underset{\alpha}{\arg \min } Y_{N}^{T}\left(\Phi_{N}^{T} P(\alpha) \Phi_{N}+\sigma^{2} I_{N}\right)^{-1} Y_{N} \\
& \quad+\log \left|\Phi_{N}^{T} P(\alpha) \Phi_{N}+\sigma^{2} I_{N}\right|
\end{aligned}
$$

where $|\cdot|$ denotes the determinant of a matrix and $I_{N}$ denotes the $N$-dimensional identity matrix. This method is widely known as the empirical Bayes method (Carlin \& Louis, 1996). It is the maximum likelihood method to estimate $\alpha$ from (4) under the (Bayesian) assumptions that $\theta$ is Gaussian with zero mean and covariance matrix $P(\alpha)$ and $V_{N}$ is Gaussian with zero mean and covariance matrix $\sigma^{2} I_{N}$. The parameter vector $\alpha$ is typically called hyper-parameter vector in the Bayesian setting and the prior covariance matrix $P(\alpha)$ is also often called the kernel matrix in machine learning, e.g. (Rasmussen \& Williams, 2006).

The regularized least squares (5) including the empirical Bayes method (6), has been proven very successful in many different scenarios, like the Bayesian inference, e.g. (Carlin \& Louis, 1996), machine learning, e.g. (Rasmussen \& Williams, 2006) and system identification, e.g. (Pillonetto \& Nicolao, 2010; Pillonetto et al., 2011; Chen, Ohlsson \& Ljung, 2012). One key of this approach lies in solving the marginal likelihood maximization problem (6) that is usually nonconvex. No matter what nonconvex optimization solver is used, the cost function in (6) has to be computed repetitively for different $\alpha$. Various methods to compute the cost function in (6) (also the estimate (5b)) efficiently and accurately have been discussed extensively in machine learning, e.g. (Rasmussen \& Williams, 2006; QuiñoneroCandela, Rasmussen \& Williams, 2007). However, those methods in (Rasmussen \& Williams, 2006; QuiñoneroCandela et al., 2007) may not be well applied to the scenario of system identification because the considerations are very different in machine learning and system identification. For example, it is usually assumed $n \gg N$ in Gaussian process regression (Rasmussen \& Williams, 2006) but it is typically assumed $N \gg n$ in system identification since it is preferable to have much more data points than the number of model parameters. That has prompted the current wish to study efficient and accurate algorithms to compute the cost function in (6) in the scenario of system identification. In particular, there are two major issues regarding the computation of the cost function in (6):

\section{- computational complexity}

The computational complexity of the cost function in (6) is determined by three integers, $N$ (the number of observations used), $n$ (the order of the FIR model (3)), $m$ (the dimension of $\alpha$ ) and the parameterization of the covariance matrix $P(\alpha)$. For the impulse response estimation, $m$ often is small, $2-4$ or so, the number of parameters $n$ is typically quite large, a couple of hundred or so, and the algorithm should be able to deal with very large number of data $N$, certainly several thousands. We may note that the matrix inverse in (6) is of size $N \times N$ and thus has computational complexity of $O\left(N^{3}\right)$. So straightforward computation of the cost function in (6) can be very timeconsuming for very large $N$. Here, we will focus on how to compute the cost function in (6) efficiently with very large $N$ and $N \gg n$.

- numerical accuracy

When seeking efficient algorithms to compute the cost function in (6) for very large $N$ and $N \gg n$, the numerical accuracy depends on the conditioning and the magnitude of the matrices $P(\alpha)$ and $\Phi_{N} \Phi_{N}^{T}$. Both $P(\alpha)$ and $\Phi_{N} \Phi_{N}^{T}$ can be ill-conditioned and moreover, have very large magnitude compared to the noise level $\sigma^{2} I_{n}$. They may cause problems when computing the cost function in (6), which will be made clear in Section 2. So computation of the cost function in (6) (also the estimate (5b)) needs to be numerically accurate to handle these problems.

In the context of system identification, the numerical accuracy issue has not been studied before, but the computational complexity issue was recently studied in (Carli, Chiuso \& Pillonetto, 2012). The proposed approach therein, however, only works for the family of so-called stable spline kernels (Pillonetto \& Nicolao, 2010) but not the other kernels for impulse response estimation (Chen, Ohlsson \& Ljung, 2012; Pillonetto \& Nicolao, 2011; Chen, Ohlsson \& Ljung, 2011) (See Remark 2.3 for more detailed discussions).

In this paper, we investigate implementation of algorithms to compute the cost function in (6) that can handle the two issues aforementioned. In particular, we propose a QR factorization based matrix-inversion-free algorithm. It is shown to have the computational complexity of $O\left(n^{3}\right)$ and to be more accurate than a modification of Algorithm 2.1 in (Rasmussen $\&$ Williams, 2006). We further show the gradient and Hessian of the cost function in (6) can be computed based on the same QR factorization. Finally, we verify the proposed algorithm and ideas by Monte-Carlo simulations on the databank used in (Chen, Ohlsson \& Ljung, 2012).

\section{A straightforward implementation}

By exploiting the structure of the cost function in (6), we can derive an efficient way to compute it accordingly, which can be seen as a straightforward modification of Algorithm 2.1 in (Rasmussen \& Williams, 2006). By matrix inversion lemma and Sylvester's determinant theorem, e.g., (Harville, 2008), we can rewrite the cost function in (6) so that recomputation of the cost function in (6) for new values of $\alpha$ have a complexity independent of $N$.

The cost function in (6) is for convenience copied below 


$$
Y_{N}^{T}\left(\Phi_{N}^{T} P(\alpha) \Phi_{N}+\sigma^{2} I_{N}\right)^{-1} Y_{N}+\log \left|\Phi_{N}^{T} P(\alpha) \Phi_{N}+\sigma^{2} I_{N}\right|
$$

Let us begin with some reformulations of (7). By Sylvester's determinant theorem e.g., (Harville, 2008),

$$
\begin{aligned}
& \log \left|\sigma^{2} I_{N}+\Phi_{N}^{T} P(\alpha) \Phi_{N}\right|=(N-n) \log \sigma^{2} \\
& \quad+\log |P(\alpha)|+\log \left|\sigma^{2} P(\alpha)^{-1}+\Phi_{N} \Phi_{N}^{T}\right|
\end{aligned}
$$

On the other hand, by the matrix inversion lemma,

$$
\begin{aligned}
& Y_{N}^{T}\left(\sigma^{2} I_{N}+\Phi_{N}^{T} P(\alpha) \Phi_{N}\right)^{-1} Y_{N}=Y_{N}^{T} Y_{N} / \sigma^{2} \\
& \quad-Y_{N}^{T} \Phi_{N}^{T}\left(\sigma^{2} P(\alpha)^{-1}+\Phi_{N} \Phi_{N}^{T}\right)^{-1} \Phi_{N} Y_{N} / \sigma^{2}
\end{aligned}
$$

It can be seen from (8) and (9) that the computational complexity of (7) now becomes independent of $N$ if the scalar $\left\|Y_{N}\right\|^{2}$, the $n \times n$ matrix $\Phi_{N} \Phi_{N}^{T}$ and the $n \times 1$ column vector $\Phi_{N} Y_{N}$ are computed and saved beforehand.

Nevertheless, (8) and (9) need to find $P(\alpha)^{-1}$, which we should avoid to compute directly. This is because $P(\alpha)$ is often ill-conditioned for the impulse response estimation. This point will be made clear shortly. Note that with the Cholesky factorization of $P(\alpha)$ :

$$
P(\alpha)=L L^{T}
$$

(8) and (9) can be put into the form

$$
\begin{aligned}
& \log \left|\sigma^{2} I_{N}+\Phi_{N}^{T} P(\alpha) \Phi_{N}\right|= \\
& (N-n) \log \sigma^{2}+\log \left|\sigma^{2} I_{n}+L^{T} \Phi_{N} \Phi_{N}^{T} L\right| \\
& Y_{N}^{T}\left(\sigma^{2} I_{N}+\Phi_{N}^{T} P(\alpha) \Phi_{N}\right)^{-1} Y_{N}= \\
& Y_{N}^{T} Y_{N} / \sigma^{2}-Y_{N}^{T} \Phi_{N}^{T} L\left(\sigma^{2} I_{n}+L^{T} \Phi_{N} \Phi_{N}^{T} L\right)^{-1} L^{T} \Phi_{N} Y_{N} / \sigma^{2}
\end{aligned}
$$

In this way, we have avoided to compute $P(\alpha)^{-1}$. Then, in light of Algorithm 2.1 of (Rasmussen \& Williams, 2006), we have the following way to compute the cost function in (6).

Algorithm 1 Assume $\left\|Y_{N}\right\|^{2}, \Phi_{N} \Phi_{N}^{T}$ and $\Phi_{N} Y_{N}$ are computed and saved. The algorithm consists of the following steps to compute the cost function (7) in (6):

1) compute the Cholesky factorization L of $P(\alpha)$

2) compute $\sigma^{2} I_{n}+L^{T} \Phi_{N} \Phi_{N}^{T} L$

3) compute the Cholesky factorization of $\sigma^{2} I_{n}+L^{T} \Phi_{N} \Phi_{N}^{T} L$ :

$$
\sigma^{2} I_{n}+L^{T} \Phi_{N} \Phi_{N}^{T} L=S S^{T}
$$

4) compute $S^{-1}$
5) compute $\left\|Y_{N}\right\|^{2}-\left\|S^{-1} L^{T} \Phi_{N} Y_{N}\right\|^{2}$

6) compute $\left(\left\|Y_{N}\right\|^{2}-\left\|S^{-1} L^{T} \Phi_{N} Y_{N}\right\|^{2}\right) / \sigma^{2}+(N-n) \log \sigma^{2}$ $+2 \log |S|$

Accordingly, the regularized least-squares estimate $\hat{\theta}_{N}$ can be computed according to $\hat{\theta}_{N}=L S^{-T} S^{-1} L^{T} \Phi_{N} Y_{N}$.

Remark 2.1 In practice, the noise variance $\sigma^{2}$ is not known and thus needs to be estimated from the data. As suggested in (Goodwin, Gevers \& Ninness, 1992; Ljung, 1999), a simple and effective way to estimate $\sigma^{2}$ is to first estimate an ARX model (Pillonetto \& Nicolao, 2010; Pillonetto et al., 2011) or an FIR model (Chen, Ohlsson \& Ljung, 2012) with least squares and then choose the sample variance as the estimate of the noise variance. There are standard and efficient routines to accomplish this task, so we will not discuss how to estimate $\sigma^{2}$ here and moreover, we will assume $\sigma^{2}$ is known. Instead of the way mentioned above, we can also treat $\sigma^{2}$ as another "hyper-parameter" and estimate it together with $\alpha$. It is straightforward to see all the following arguments with minor changes also apply to this case.

Remark 2.2 The technique of applying the Cholesky factorization method in steps 3) to 5) of Algorithm 1 to compute the cost function in (6) is not new and actually widely used in machine learning, see e.g. (Rasmussen \& Williams, 2006). Moreover, by exploiting the structure of the cost function in (6) and the Cholesky factorization of $P(\alpha)$ (10), Algorithm 1 can be seen as a straightforward modification of Algorithm 2.1 in (Rasmussen \& Williams, 2006). Algorithm 2.1 in (Rasmussen \& Williams, 2006) is however not suited to the case studied here because it has a computational complexity of $O\left(N^{3}\right)$ (note that it assumes $n \gg N$ ).

Remark 2.3 For large data sets (very large $N$ ), various methods to compute the cost function in (6) have been studied in machine learning, e.g. (Rasmussen \& Williams, 2006, Chapter 8) and (Quiñonero-Candela et al., 2007). However, those methods may not be well applied to the scenario of system identification because the considerations are very different in machine learning and system identification. One exception is (Carli et al., 2012) where the infinite impulse response of linear stable systems is modeled as a zero mean Gaussian process with the so-called stable spline kernel (Pillonetto \& Nicolao, 2010). Instead of truncating the infinite impulse response to a finite one, the algorithm in (Carli et al., 2012) truncates the kernel representations of the stable spline kernel (Pillonetto \& Bell, 2007) and can efficiently compute the cost function in (6) (also the estimate (5b)). Its computational complexity scales as $O\left(l^{3}\right)$ where $l$ is the number of truncated eigenfunctions. Comparing Algorithm 1 and the algorithm in (Carli et al., 2012), it is problem dependent regarding which one is more efficient (see (Carli et al., 2012, p. 5) for relevant discussions). However, the algorithm in (Carli et al., 2012) only works for the family of stable spline kernels but not the other kernels for impulse response estimation (Chen, Ohlsson \& Ljung, 2012; Pillonetto \& Nicolao, 2011; Chen et al., 2011). Moreover, the 


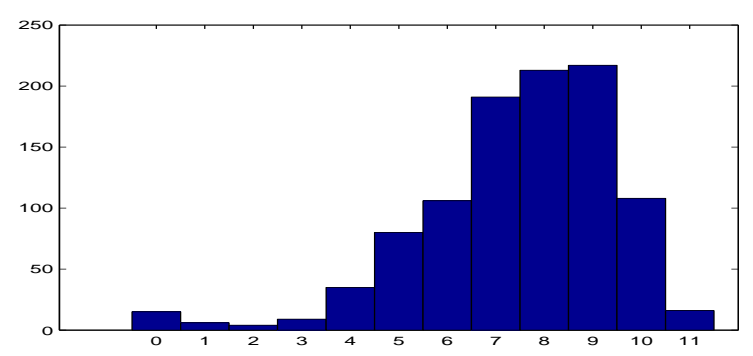

Fig. 1. Histogram-plot of $\log _{10} \operatorname{cond}\left(\sigma^{2} I_{n}+L^{T} \Phi_{N} \Phi_{N}^{T} L\right)$ over 1000 data sets in the second experiment of (Pillonetto \& Nicolao, 2011; Chen et al., 2011). Here, we consider the stable spline kernel (Pillonetto \& Nicolao, 2010) with the optimal hyper-parameter estimate $\hat{\alpha}$ from solving (6).

numerical accuracy issue due to the possibly ill-conditioned $\Phi_{N}^{T} \Phi_{N}$ and $P(\alpha)$ is not addressed in (Carli et al., 2012).

Now we check the numerical accuracy of Algorithm 1. For given $\Phi_{N}, Y_{N}$ and $L$, we find computation of the term in (12)

$$
\left(\sigma^{2} I_{n}+L^{T} \Phi_{N} \Phi_{N}^{T} L\right)^{-1} L^{T} \Phi_{N} Y_{N}
$$

is important for the accuracy. Note that (14) can be seen as the solution of the following least squares problem

$$
\min _{x}|| A x-b \|^{2}
$$

where $x \in \mathbb{R}^{n}$ is the unknown,

$$
A=\left[\begin{array}{c}
\Phi_{N}^{T} L \\
\sigma
\end{array}\right], \quad b=\left[\begin{array}{c}
Y_{N} \\
0
\end{array}\right]
$$

Actually, the steps 3) to 5) of Algorithm 1 implicitly solves (15) with the Cholesky factorization method (also known as the method of normal equations, see e.g. (Golub \& Van Loan, 1996, Algorithm 5.3.1)) and gives the solution as $S^{-T} S^{-1} L^{T} \Phi_{N} Y_{N}$. It is well-known, e.g. (Golub \& Van Loan, 1996), that the Cholesky factorization method to the least squares problem (15) is not accurate especially when $A^{T} A$ is ill-conditioned.

For the impulse response estimation problem, the matrix

$$
A^{T} A=\sigma^{2} I_{n}+L^{T} \Phi_{N} \Phi_{N}^{T} L
$$

can be ill-conditioned due to the following two problems:

- The matrix $L^{T} \Phi_{N} \Phi_{N}^{T} L$ can be very ill-conditioned.

On the one hand, $P(\alpha)$ is typically assumed to have an exponentially decaying diagonal and thus $P(\alpha)$ (so is $L$, recall (10)) can be ill-conditioned if the element of $\alpha$ that controls the decaying rate is very small. For example, consider the "DC" $\operatorname{kernel}(31 \mathrm{c})$ where $\alpha=\left[\begin{array}{ccc}c & \lambda & \rho\end{array}\right]^{T}$, $c \geq 0$ controls the magnitude of $P(\alpha), 0 \leq \lambda \leq 1$ controls the decay of $P(\alpha)$ and $|\rho| \leq 1$ controls the correlation between the impulse response coefficients. For the case $n=125, \operatorname{cond}(P(\alpha))$ (independent of $c$ ) is $2.99 \times 10^{8}$ for $\lambda=0.9, \rho=0.98$ and $3.84 \times 10^{29}$ for $\lambda=0.6, \rho=0.98$. Here, $\operatorname{cond}(\cdot)$ is used to denote the 2-norm condition number of a matrix and is computed with the command cond in Matlab.

On the other hand, the matrix $\Phi_{N} \Phi_{N}^{T}$ can be illconditioned too. For example, consider the band-limited input case (Pillonetto et al., 2011; Pillonetto \& Nicolao, 2011; Chen et al., 2011). Set $N=500$ and $n=125$, and generate (using the command idinput $(500$, ' rgs', $\left[\begin{array}{ll}0 & 0.8\end{array}\right]$ ) in Matlab) the Gaussian random signal with the frequency band $\left[\begin{array}{ll}0 & 0.8\end{array}\right]$ where 0 and 0.8 are the lower and upper limits of the passband, expressed in fractions of the Nyquist frequency. For the instance we tried, $\operatorname{cond}\left(\Phi_{N} \Phi_{N}^{T}\right)$ is $3.63 \times 10^{13}$.

- Second, the magnitude of $\sigma^{2} I_{n}$ can be very small compared to that of $L^{T} \Phi_{N} \Phi_{N}^{T} L$. In this case, $\sigma^{2} I_{n}+$ $L^{T} \Phi_{N} \Phi_{N}^{T} L$ can be ill-conditioned if $L^{T} \Phi_{N} \Phi_{N}^{T} L$ is very ill-conditioned. The magnitude of $L^{T} \Phi_{N} \Phi_{N}^{T} L$ can be very large if the element of $\alpha$ that controls the magnitude of $P(\alpha)$ is large, which is often the case for the stable spline kernel (Pillonetto \& Nicolao, 2010; Pillonetto et al., 2011). As for the magnitude of $\Phi_{N} \Phi_{N}^{T}$, it can also be large because it depends on the input asserted to the system to be identified.

For illustration, consider the second experiment of (Pillonetto \& Nicolao, 2011; Chen et al., 2011) which contains 1000 data sets. Moreover, consider the stable spline kernel (Pillonetto \& Nicolao, 2010) with the optimal hyperparameter estimate $\hat{\alpha}$ from solving (6). It can then be seen from the histogram plot of $\log _{10} \operatorname{cond}\left(\sigma^{2} I_{n}+L^{T} \Phi_{N} \Phi_{N}^{T} L\right)$ over 1000 data sets in the second experiment of (Pillonetto $\&$ Nicolao, 2011; Chen et al., 2011) in Fig. 1 that, the corresponding $\sigma^{2} I_{n}+L^{T} \Phi_{N} \Phi_{N}^{T} L$ is often ill-conditioned.

The above observations motivate the need to find a numerically more accurate implementation than Algorithm 1.

\section{When QR factorization meets the marginal likeli- hood maximization}

It is well-known, e.g. (Golub \& Van Loan, 1996, Section 5), that the least squares problem (15) can be solved more accurately with the $\mathrm{QR}$ factorization method than the Cholesky factorization method, when the condition number of $A^{T} A$ defined in (17) is ill-conditioned. In this section, we show the cost function in (6) can be computed with the help of QR factorizations.

Before we proceed, first recall the definition of thin QR factorization cf. (Golub \& Van Loan, 1996, p. 230): if $B=C D$ is a QR factorization of $B \in \mathbb{R}^{p \times q}$ with $p \geq q$, then $B=C$ (: $, 1: q) D(1: q, 1: q)$ is referred to as the thin QR factorization of $B$. Here, $C(:, 1: q)$ is the matrix consisting of the first 
$q$ columns of $C$ and $D(1: q, 1: q)$ is the matrix consisting of the first $q$ rows and $q$ columns of the matrix $D$. We then make two assumptions to guarantee the uniqueness of the thin QR factorization in the following, cf. (Golub \& Van Loan, 1996, p. 230, Thm 5.2.2).

1) Without loss of generality, assume

$$
\operatorname{rank}\left[\Phi_{N}^{T} Y_{N}\right]=n+1
$$

2) Assume all upper triangular matrices involved in the thin QR factorizations below have positive diagonal entries.

Now perform the thin QR factorization of

$$
\left[\begin{array}{cc}
\Phi_{N}^{T} L & Y_{N} \\
\sigma I_{n} & 0
\end{array}\right]=Q R=Q\left[\begin{array}{cc}
R_{1} & R_{2} \\
0 & r
\end{array}\right]
$$

where $Q$ is a $(N+n) \times(n+1)$ matrix whose columns are orthogonal unit vectors such that $Q^{T} Q=I_{n+1}$, and $R$ is an $(n+1) \times(n+1)$ upper triangular matrix. Here, $R$ is further partitioned into $2 \times 2$ blocks with $R_{1}, R_{2}$ and $r$ being a $n \times n$ matrix, a $n \times 1$ vector and a scalar, respectively.

Now noting $Q^{T} Q=I_{n+1}$ yields that

$$
\begin{aligned}
& \sigma^{2} I_{n}+L^{T} \Phi_{N} \Phi_{N}^{T} L=R_{1}^{T} R_{1} \\
& L^{T} \Phi_{N} Y_{N}=R_{1}^{T} R_{2} \\
& Y_{N}^{T} Y_{N}=R_{2}^{T} R_{2}+r^{2}
\end{aligned}
$$

Therefore, the right-hand side of (11) can be computed as

$$
\begin{aligned}
& (N-n) \log \sigma^{2}+\log \left|\sigma^{2} I_{n}+L^{T} \Phi_{N} \Phi_{N}^{T} L\right| \\
& =(N-n) \log \sigma^{2}+\log \left|R_{1}\right|^{2}
\end{aligned}
$$

and the right-hand side of (12) can be computed as

$$
\begin{aligned}
& Y_{N}^{T} Y_{N} / \sigma^{2}-Y_{N}^{T} L \Phi_{N}^{T}\left(\sigma^{2} I_{n}+L^{T} \Phi_{N} \Phi_{N}^{T} L\right)^{-1} L^{T} \Phi_{N} Y_{N} / \sigma^{2} \\
& =\left(R_{2}^{T} R_{2}+r^{2}\right) / \sigma^{2}-R_{2}^{T} R_{1}\left(R_{1}^{T} R_{1}\right)^{-1} R_{1}^{T} R_{2} / \sigma^{2} \\
& =r^{2} / \sigma^{2}
\end{aligned}
$$

where the fact that $R_{1}$ is nonsingular has been used in deriving the second equation.

As a result, we have the following proposition regarding how to compute (7), i.e. the cost function in (6).

Proposition 3.1 Consider (7), i.e., the cost function in (6). Assume the thin QR factorization (19) has been computed, and $R_{1}, R_{2}, r$ are available. The cost function (7) in (6) can be computed according to

$$
\begin{aligned}
& Y_{N}^{T}\left(\Phi_{N}^{T} P(\alpha) \Phi_{N}+\sigma^{2} I_{N}\right)^{-1} Y_{N}+\log \left|\Phi_{N}^{T} P(\alpha) \Phi_{N}+\sigma^{2} I_{N}\right| \\
& =r^{2} / \sigma^{2}+(N-n) \log \sigma^{2}+2 \log \left|R_{1}\right|
\end{aligned}
$$

Moreover, the regularized FIR estimate (5) for the given $\alpha$ can be computed according to

$$
\hat{\theta}_{N}=L R_{1}^{-1} R_{2}
$$

Now we check the numerical accuracy of Algorithm 2 and consider the computation of (14) in (22), i.e., the solution of the least squares problem (15) again. As can be seen from Proposition 3.1, the least squares problem (15) is solved with QR factorization based method. More specifically, consider the QR factorization of

$$
\left[\begin{array}{ll}
A & b
\end{array}\right]=\left[\begin{array}{cc}
\Phi_{N}^{T} L & Y_{N} \\
\sigma I_{n} & 0
\end{array}\right]=\bar{Q} \bar{R}
$$

where $A, b$ are defined in (16), $\bar{Q}$ is a $(N+n) \times(N+n)$ orthogonal matrix and $\bar{R}$ is a $(N+n) \times(n+1)$ upper triangular matrix. Note that $Q$ in (19) is the block matrix consisting of the first $n+1$ columns of $\bar{Q}$ and $R$ in (19) is the block matrix consisting of first $n+1$ rows of $\bar{R}$. From the QR factorization (25) and (19), the least squares problem (15) becomes

$$
\begin{aligned}
\min _{x}\|A x-b\|^{2}=\min _{x}\left\|\bar{Q}^{T} A x-\bar{Q}^{T} b\right\|^{2} \\
=\min _{x}\left\|\left[\begin{array}{c}
R_{1} \\
0 \\
0
\end{array}\right] x-\left[\begin{array}{c}
R_{2} \\
r \\
0
\end{array}\right]\right\|^{2}=\min _{x}\left\|R_{1} x-R_{2}\right\|^{2}+r^{2}
\end{aligned}
$$

which yields the optimal $x^{*}=\left(R_{1}^{T} R_{1}\right)^{-1} R_{1}^{T} R_{2}=R_{1}^{-1} R_{2}$.

This way of solving the least squares problem (15) is standard, (Golub \& Van Loan, 1996, Section 5.3.3), and known, (Golub \& Van Loan, 1996, Section 5.3.8), to be more accurate than the Cholesky factorization method used in Algorithm 1 , when $A^{T} A$ defined in (17) is ill-conditioned. In this sense, we have obtained a numerically more accurate way to compute the cost function (7) in (6).

On the other hand, let us consider the computational complexity issue. We can of course compute the cost function (7) according to Proposition 3.1. Clearly, the major computation cost relies on the QR factorization (19), which is dependent on $N$. It is however worthwhile to note that $\Phi_{N}^{T}$ and $Y_{N}$ are fixed when solving the marginal likelihood maximization problem (6) and the only varying thing is $P(\alpha)$ (and thus $L$ in (10)). So an interesting question is if this observation can be made use of to compute the QR factorization (19) in 
a more efficient way (to make the computational complexity independent of $N$ ).

The answer to the above question is definite. To see this, let us consider the thin $\mathrm{QR}$ factorization of the matrix

$$
\left[\begin{array}{ll}
\Phi_{N}^{T} & Y_{N}
\end{array}\right]=Q_{d}\left[\begin{array}{ll}
R_{d 1} & R_{d 2}
\end{array}\right]
$$

where $Q_{d}$ is an $N \times(n+1)$ matrix whose columns are orthogonal unit vectors such that $Q_{d}^{T} Q_{d}=I_{n+1}, R_{d 1}$ is a $(n+$ $1) \times n$ matrix and $R_{d 2}$ is a $(n+1) \times 1$ vector.

Now consider further the thin QR factorization of

$$
\left[\begin{array}{cc}
R_{d 1} L & R_{d 2} \\
\sigma I_{n} & 0
\end{array}\right]=Q_{c} R_{c}
$$

where $Q_{c}$ is an $(2 n+1) \times(n+1)$ matrix whose columns are orthogonal unit vectors such that $Q_{c}^{T} Q_{c}=I_{n+1}$ and $R_{c}$ is a $(n+1) \times(n+1)$ upper triangular matrix.

Then from (27) and (28), we have

$$
\left[\begin{array}{cc}
\Phi_{N}^{T} L & Y_{N} \\
\sigma I_{n} & 0
\end{array}\right]=\left[\begin{array}{cc}
Q_{d} & 0 \\
0 & I_{n}
\end{array}\right] Q_{c} R_{c}
$$

Noting the two assumptions mentioned in the beginning of this section and positive definite $L$, comparing (19) with (29) and invoking the uniqueness of the thin QR factorization (Golub \& Van Loan, 1996, p. 230, Thm 5.2.2) yields that $R$ and $Q$ in (19) can be computed according to

$$
R=R_{c}, \quad Q=\left[\begin{array}{cc}
Q_{d} & 0 \\
0 & I_{n}
\end{array}\right] Q_{c}
$$

In this way (using (28) and (30)), we find a more efficient way to compute the QR factorization (19) and get the following algorithm to compute the cost function (7) in (6).

Algorithm 2 Assume the thin $Q R$ factorization (27) has been computed, and $R_{d 1}, R_{d 2}$ are available. The algorithm consists of the following steps to compute the cost function (7) in (6):
1) compute the Cholesky factorization $L$ of $P(\alpha)$
2) compute $R_{d 1} L$
3) compute the $Q R$ factorization (28)
4) compute $r^{2} / \sigma^{2}+(N-n) \log \sigma^{2}+2 \log \left|R_{1}\right|$

Remark 3.1 It is worthwhile to note that it is not necessary to compute the " $Q$ " matrix in all thin $Q R$ factorizations aforementioned, because it is not used in Algorithm 2. In Matlab, the command triu ( qr (·)) can be used to derive the $Q R$ factorization without computing the " $Q$ " matrix.
Remark 3.2 From (13) and (20a), $\sigma^{2} I_{n}+L^{T} \Phi_{N} \Phi_{N}^{T} L=$ $S S^{T}=R_{1}^{T} R_{1}$. If the Cholesky factorization $S$ is assumed to have positive diagonal entries, then we have $S=R_{1}^{T}$ in theory. Actually, (19) shows one typical way to compute the Cholesky factorization of $\sigma^{2} I_{n}+L^{T} \Phi_{N} \Phi_{N}^{T} L$ (Golub \& Van Loan, 1996, p. 230). When analyzing the computational accuracy issue in Section 4.1, we will assume $S=R_{1}^{T}$.

\section{Computational accuracy and complexity}

In this section, let us go back to the two implementation issues mentioned in the Introduction and check the two algorithms accordingly.

\subsection{Computational accuracy}

For convenience, the accuracy issue has been discussed in the text in a distributed manner. Here we will give a brief summary to highlight our findings.

For given $\Phi_{N}, Y_{N}$ and $L$ and with the assumption $S=R_{1}^{T}$, comparing (11) and (13) with (21) shows the computation of Algorithms 1 and 2 only differs in (12) and more specifically, it only differs in (14), i.e., the solution of the least squares problem (15), which becomes critical for comparison of the two algorithms in computational accuracy.

As mentioned in Sections 2 and 3, both of the two algorithms solve the least squares problem (15) implicitly:

- As can be seen from steps 3) to 5), (15) is solved in Algorithm 1 with the Cholesky factorization method, i.e., (Golub \& Van Loan, 1996, Algorithm 5.3.1).

- As can be seen from (22), (15) is solved in Algorithm 2 with the QR factorization method in (Golub \& Van Loan, 1996, Section 5.3.3).

It is known from (Golub \& Van Loan, 1996, Section 5.3.8) and the references therein that for ill-conditioned $A^{T} A$, compared to the Cholesky factorization method the QR factorization method can yield more accurate solution to the least squares problem (15), i.e. more accurate (14). Therefore, we conclude Algorithm 2 gives more accurate computation of the cost function in (6) than Algorithm 1. Also note that with (10), (5b) becomes $\hat{\theta}_{N}=L\left(\sigma^{2} I_{n}+L^{T} \Phi_{N} \Phi_{N}^{T} L\right)^{-1} L^{T} \Phi_{N} Y_{N}$. Accordingly, Algorithm 2 can also be used to yield more accurate computation of $\hat{\theta}_{N}$ than Algorithm 1.

\subsection{Computational complexity}

Both of the two algorithms consist of two parts: the preprocessing and the evaluation of the cost function (7) in (6). So let us consider the two parts separately:

- preprocessing 
For Algorithm 1, the scalar $\left\|Y_{N}\right\|^{2}$, the $n \times n$ matrix $\Phi_{N} \Phi_{N}^{T}$ and the $n \times 1$ column vector $\Phi_{N} Y_{N}$ need to be computed beforehand. They require $2 N-1, n^{2} N+n N-$ $N^{2} / 2-N / 2$ and $2 N n-n$ flops, respectively. For Algorithm 2, the QR factorization (27) needs to be computed beforehand and requires $2(n+1)^{2}(N-(n+1) / 3)$ flops according to (Golub \& Van Loan, 1996).

- evaluation of the cost function (7) in (6)

First note that the computational complexity of the steps 1) and 6) of Algorithm 1 is same as that of the steps 1) and 4) of Algorithm 2. According to (Hunger, 2007), they require $n^{3} / 3+n^{2} / 2+n / 6$ and $2 n+6$ flops, respectively. The steps 2) to 5) of Algorithm 1 requires $2 n^{3}+n$, $n^{3} / 3+n^{2} / 2+n / 6, n^{3} / 3+2 n / 3$, and $2 n^{2}+2 n$ flops, respectively. The step 2) of Algorithm 2 requires $n^{2}(n+1)$ flops and straightforward computation of the $\mathrm{QR}$ factorization (28) requires $2(n+1)^{2}(2 n+1-(n+1) / 3)$ flops according to (Golub \& Van Loan, 1996).

For both of the two parts, we see Algorithm 2 requires more flops (about twice) than Algorithm 1.

\subsection{Recommended algorithm}

The FIR model (3) order $n$ is assumed to be reasonably large here (typically a couple of hundred or so). For such size problems, the factor of about two in the computational complexity does not outweigh the difference in the computational accuracy, so Algorithm 2 is the recommended algorithm.

Remark 4.1 If the impulse response is decaying slowly, a very high order FIR model (with very large $n$ ) will be required. In this case, as discussed in (Chen, Ohlsson \& Ljung, 2012), we can first estimate, with the Maximum Likelihood/Prediction Error Method e.g. (Ljung, 1999), a low-order "base-line model" that can take care of the dominating part of the impulse response. We then use regularized least squares (based on Algorithm 2) to estimate an FIR model with reasonably large $n$, which should capture the residual (fast decaying) dynamics (Chen, Ohlsson \& Ljung, 2012).

Remark 4.2 Both Algorithms 1 and 2 are based on the Cholsesky factorization of the kernel matrix $P(\alpha)$. However, what really matters is that $P(\alpha)$ can be decomposed into the form of $P(\alpha)=L L^{T}$ where $L$ is not necessarily its Cholesky factorization. This means it is possible to employ other decompositions of $P(\alpha)$ to compute $L$ in both Algorithms 1 and 2, for example, the singular value decomposition (SVD). Actually, SVD of the kernel matrix has been used in machine learning, see e.g., (Pelckmans, Brabanter, Suykens \& Moor, 2005; Pelckmans, Suykens \& Moor, 2006). Note that the SVD of $P(\alpha): P(\alpha)=U F U^{T}=U F^{1 / 2} F^{1 / 2} U^{T}$ where $U \in \mathbb{R}^{n \times n}$ is an orthogonal matrix, $F$ is a diagonal matrix whose diagonal entries are singular values of $P(\alpha)$ and $F^{1 / 2}$ is the square root of $F$. Then $L$ in Algorithm 1 can be computed as $L=U F^{1 / 2}$. In contrast with the computation of $L$ via Cholesky factorization, the computation of $L$ via SVD requires $12 n^{3}+n^{2}+n$ flops on average (Golub \& Van Loan, 1996, p. 254), about 36 times more.

Remark 4.3 For impulse response estimation problems, $P(\alpha)$ can often have very large condition number, which can cause numerical problems, for example, the failure or inaccuracy of the Cholesky factorization of $P(\alpha)$ in (10). Of course, one may look through the literature of numerical computation, e.g. (Golub \& Van Loan, 1996) and find stable and accurate ways to compute the Cholesky factorization or alternative decompositions that can be used for Algorithms 1 and 2. Here, we however tackle this problem in an "active" way based on our knowledge for $P(\alpha)$ in the impulse response estimation.

As shown in Section 2, the kernel $P(\alpha)$ can have very large condition number if the element of $\alpha$ that controls the decaying rate of $P(\alpha)$ is very small. Having this observation in mind, it is natural to actively choose to enforce extra constraint on $\alpha$ to guarantee $P(\alpha)$ has a tolerably large condition number so as to avoid numerical problems. For illustration, consider FIR model (3) with $n=125$ and the four kernels studied in (Chen, Ohlsson \& Ljung, 2012; Pillonetto \& Nicolao, 2011; Chen et al., 2011):

$$
\begin{aligned}
& P_{i, j}^{D I}(\alpha)=\left\{\begin{array}{c}
c \lambda^{k} \text { if } k=j, \quad \alpha=\left[\begin{array}{ll}
c & \lambda
\end{array}\right]^{T} \\
0 \text { else }
\end{array}\right. \\
& P_{i, j}^{T C}(\alpha)=c \min \left(\lambda^{j}, \lambda^{k}\right), \quad \alpha=\left[\begin{array}{ll}
c & \lambda
\end{array}\right]^{T} \\
& P_{i, j}^{D C}(\alpha)=c \rho^{|k-j|} \lambda^{(k+j) / 2}, \quad \alpha=\left[\begin{array}{lll}
c & \lambda & \rho
\end{array}\right]^{T} \\
& P_{i, j}^{S S}(\alpha)=\left\{\begin{array}{ll}
c \frac{\lambda^{2 i}}{2}\left(\lambda^{j}-\frac{\lambda^{i}}{3}\right), i \geq j \\
c \frac{\lambda^{2 j}}{2}\left(\lambda^{i}-\frac{\lambda^{j}}{3}\right), i<j
\end{array}, \quad \alpha=[c, \lambda]^{T}\right.
\end{aligned}
$$

where we usually have $c \geq 0,0 \leq \lambda<1,|\rho| \leq 1$. Now, we impose the following extra constraints:

- For the "DI" kernel (31a) and "TC" kernel (31b), further assume $0.7 \leq \lambda<1$ so that the condition number of the DI kernel is smaller than $1.6 \times 10^{19}$ and that of the TC kernel is smaller than $2.4 \times 10^{20}$.

- For the "DC" kernel (31c), further assume $0.72 \leq \lambda<1$ and $-0.99 \leq \rho \leq 0.99$ so that the condition number of the DC kernel is smaller than $2.0 \times 10^{20}$.

- For the "SS" kernel (31d), further assume $0.9 \leq \lambda<1$ so that the condition number of the SS kernel is smaller than $1.9 \times 10^{21}$.

The extra constraints limit the search region of $\alpha$ for (6). So one may question if the extra constraints can eventually cause performance loss in the regularized least squares estimate $(5 b)$. Our answer to this question is that at least for the large test data-bank in (Chen, Ohlsson \& Ljung, 2012), the Monte Carlo simulation results show that there is no performance loss with the above imposed extra constraints; see Section 6 for more details. 


\section{Computation of the Gradient and Hessian}

For the impulse response estimation, the dimension of the hyper-parameter $\alpha$ is often small, $2-4$, (Pillonetto \& Nicolao, 2011; Chen et al., 2011). So it is often quick enough to use some derivative-free nonlinear optimization solvers, such as the fminsearch in Matlab, to tackle the marginal likelihood maximization problem (6) (fminsearch is used in (Pillonetto \& Nicolao, 2010; Pillonetto et al., 2011; Chen, Ohlsson \& Ljung, 2012)). Now Algorithm 2 can be used to evaluate the cost function in (6) and then feed the result to the solver. On the other hand, there are many gradient and/or Hessian based nonlinear optimization solvers, which may be used to solve (6) in a faster way. In the following, we show how to compute the gradient of the cost function (7) in (6) based on QR factorization (28) and (30).

Let us denote the cost function (7) in (6) by $l(\alpha)$. Note that

$$
\frac{\partial l(\alpha)}{\partial \alpha_{i}}=\operatorname{Tr}\left(\frac{\partial l(\alpha)}{\partial P(\alpha)}\left(\frac{\partial P(\alpha)}{\partial \alpha_{i}}\right)^{T}\right)
$$

where $\alpha_{i}$ is the $i$ th element of $\alpha$ and $\operatorname{Tr}(\cdot)$ denotes the trace of a matrix. For simplicity, the dependency of $P(\alpha)$ on $\alpha$ and that of $\Phi_{N}$ and $Y_{N}$ on $N$ are omitted below. It follows from (7) to (9) that

$$
\begin{aligned}
& \frac{\partial l(\alpha)}{\partial P}=\frac{\partial}{\partial P}\left[\log \operatorname{det} P+\log \operatorname{det}\left(\sigma^{2} P^{-1}+\Phi \Phi^{T}\right)\right] \\
& \quad-\frac{\partial}{\partial P} Y^{T} \Phi^{T}\left(\sigma^{2} P^{-1}+\Phi \Phi^{T}\right)^{-1} \Phi Y / \sigma^{2} \\
& =P^{-1}-\sigma^{2} P^{-1}\left(\sigma^{2} P^{-1}+\Phi \Phi^{T}\right)^{-1} P^{-1}- \\
& P^{-1}\left(\sigma^{2} P^{-1}+\Phi \Phi^{T}\right)^{-1} \Phi Y Y^{T} \Phi^{T}\left(\sigma^{2} P^{-1}+\Phi \Phi^{T}\right)^{-1} P^{-1}
\end{aligned}
$$

From (33), (10) and (20), we thus have

$$
\frac{\partial l(\alpha)}{\partial \alpha_{i}}=\operatorname{Tr}\left(\left(X_{1}-X_{2}\right) \frac{\partial P}{\partial \alpha_{i}}\right)
$$

where $X_{1}=L^{-T} L^{-1}-\sigma^{2} L^{-T}\left(R_{1}^{T} R_{1}\right)^{-1} L^{-1}$ and $X_{2}=$ $L^{-T} R_{1}^{-1} R_{2} R_{2}^{T} R_{1}^{-T} L^{-1}$.

As for the computation of the Hessian of $l(\alpha)$, we only provide the result below

$$
\begin{aligned}
\frac{\partial^{2} l(\alpha)}{\partial \alpha_{i} \alpha_{j}} & =\operatorname{Tr}\left(\left(X_{1}-X_{2}\right) \frac{\partial^{2} P}{\partial \alpha_{i} \alpha_{j}}\right) \\
& +\operatorname{Tr}\left(\left(X_{1} \frac{\partial P}{\partial \alpha_{i}} X_{2}-\left(X_{1}-X_{2}\right) \frac{\partial P}{\partial \alpha_{i}} X_{1}\right) \frac{\partial P}{\partial \alpha_{j}}\right)
\end{aligned}
$$

since it may be less interesting to include the details. So if required, we can choose gradient and/or Hessian based nonlinear optimization solvers to tackle (6) with the gradient and Hessian computed according to (34) and (35).

\section{Numerical Simulations}

To test Algorithm 2 and the idea of constraining $\alpha$ mentioned in Remark 4.3, we use the data-bank in (Chen, Ohlsson \& Ljung, 2012, Section 2), which consists of four data collections:

- S1D1: fast systems, data sets with $M=500, \mathrm{SNR}=10$

- S2D1: slow systems, data sets with $M=500, \mathrm{SNR}=10$

- S1D2: fast systems, data sets with $M=375, \mathrm{SNR}=1$

- S2D2: slow systems, data sets with $M=375, \mathrm{SNR}=1$

Each collection contains 2500 randomly generated 30th order discrete-time systems and associated data sets. The fast systems have all poles inside the circle with center at the origin and radius 0.95 and the slow systems have at least one pole outside this circle. The signal to noise ratio (SNR) is defined as the ratio of the variance of the noise-free output over the variance of the white Gaussian noise. In all cases the input is Gaussian random signal with unit variance. For more details regarding the data bank, see (Chen, Ohlsson \& Ljung, 2012, Section 2).

For each data set, we aim to estimate FIR model (3) with $n=125$ using the regularized least squares (5) including the empirical Bayes method (6). We consider the kernels (31) with the extra constraint mentioned in Remark 4.3. The command fmincon is used here to solve the nonconvex optimization problem (6) with the trust region reflective algorithm selected. Algorithm 2 is chosen to compute the cost function in (6), and (34) and (35) are used to compute the corresponding gradient and Hessian, respectively. For all estimated FIR models, the model fit is defined as

$$
W=100\left(1-\left[\frac{\sum_{k=1}^{125}\left|g_{k}^{0}-\hat{g}_{k}\right|^{2}}{\sum_{k=1}^{125}\left|g_{k}^{0}-\bar{g}^{0}\right|^{2}}\right]^{1 / 2}\right), \quad \bar{g}^{0}=\frac{1}{125} \sum_{k=1}^{125} g_{k}^{0}
$$

The average model fit over the corresponding data collections is calculated and the simulation results are shown in Table 1, where an " $h$ " is appended to the name of the kernel to denote that the extra constraint is used. Comparing the simulation results with the ones reported in (Chen, Ohlsson \& Ljung, 2012, Examples 5 and 6), we find the proposed Algorithm 2 and idea of constraining $\alpha$ work well.

Table 1

Average model fit over 2500 data sets for four data collections in the data bank and for the kernels (31) with the extra constraint mentioned in Remark 4.3.

\begin{tabular}{lcccc}
\hline \hline & DIh & TCh & SSh & DCh \\
\hline \hline S1D1 & 86.8 & 90.4 & 90.5 & 90.8 \\
S2D1 & 70.0 & 78.0 & 78.0 & 78.0 \\
S1D2 & 62.2 & 72.6 & 71.2 & 73.1 \\
S2D2 & 37.2 & 60.4 & 58.9 & 60.8 \\
\hline \hline
\end{tabular}


Table 2

Average total time required to compute the cost function in (6) while solving (6) with the DC kernel (31c) over the 2500 data sets in S1D1. The unit of all figures in the table is "second". Items for Algorithms 1 and 2 takes the form of " $a+b$ ", where $a$ is the preprocessing time and $b$ is the total time to compute the reformulated cost function while solving (6).

\begin{tabular}{ccc}
\hline \hline & $n=125 N=250$ & $n=125 N=375$ \\
\hline \hline Algorithm 0 & 0.1265 & 0.3396 \\
Algorithm 1 & $0.0005+0.0446$ & $0.0008+0.0508$ \\
Algorithm 2 & $0.0010+0.0542$ & $0.0017+0.0596$ \\
\hline \hline
\end{tabular}

It may be also interesting to compare the time to compute the cost function in (6) required by straightforward computation without reformulating it (referred to as Algorithm 0 below), and Algorithms 1 and 2. So we run tests on S1D1 and record the time required to compute the cost function in (6) while solving problem (6) with the DC kernel (31c) by Algorithms 0,1 and 2. Here, we consider two cases with $n=125$, $N=250$ and $n=125, N=375$, respectively. For the former case, the first 375 data points for each data set in S1D1 are used. The simulation results are reported in Table 2. As $N$ increases, the time used by Algorithm 0 increases significantly. In contrast, the time required by both Algorithms 1 and 2 has just slight increase (about 10\%), which is actually due to that the solver needs more iterations to find the optimal solution when there are more data points.

\section{Conclusions}

The importance and usefulness to employ regularization for a better Mean Square Error fit to impulse response estimates (and thus to linear models in general) have been made very clear recently. Often high order FIR models are required for a good fit, which places a great demand on numerical efficiency and accuracy. We have here discussed the implementation issues and found that important improvements in the numerical properties are obtained by dimension reductions and factorization techniques. Also, to secure reasonable conditioning of the constructed matrices, the hyper-parameters need to be supervised and constrained.

In the 2012b version of the System Identification Toolbox, (Ljung, 2012) the command impulseest has been equipped with options for using various kernels. The implementation of the code to estimate the hyper-parameter follows the ideas of Algorithm 2, Remark 4.3 and Section 5 of this article.

\section{References}

Carli, F. P., Chiuso, A. \& Pillonetto, G. (2012). Efficient algorithms for large scale linear system identification using stable spline estimators, IFAC symposium on system identification, pp. 119-124.

Carlin, B. P. \& Louis, T. A. (1996). Bayes and Empirical Bayes methods for data analysis, Chapman \& Hall, London.
Chen, T., Ohlsson, H. \& Ljung, L. (2011). Kernel selection in linear system identification. Part II: A classical perspective, Proc. 50th IEEE Conference on Decision and Control and European Control Conference, Orlando, Florida, pp. 4326-4331.

Chen, T., Ohlsson, H. \& Ljung, L. (2012). On the estimation of transfer functions, regularizations and Gaussian processes - Revisited, Automatica 48: 1525-1535.

Chen, T., Zhao, Y. \& Ljung, L. (2012). Impulse response estimation with binary measurements: A regularized fir model approach, IFAC Symposium on System Identification, Brussels, Belgium, pp. 113-118.

Daniel, C. \& Wood, F. S. (1980). Fitting equation to data, 2nd edn, Wiely, Newyork.

Draper, N. \& Smith, H. (1981). Applied Regression Analysis, 2nd ed., Wiley, New York.

Golub, G. H. \& Van Loan, C. F. (1996). Matrix computations, Johns Hopkins studies in the mathematical sciences, Johns Hopkins University Press.

Goodwin, G. C., Gevers, M. \& Ninness, B. (1992). Quantifying the error in estimated transfer functions with application to model order selection, IEEE Trans. Automatic Control 37(7): 913-929.

Harville, D. A. (2008). Matrix Algebra From a Statistician's Perspective, Springer.

Hunger, R. (2007). Floating point operations in matrix-vector calculus, Tech. Rep. TUM-LNS-TR-05-05, Munich University of Technology .

Ljung, L. (1999). System Identification - Theory for the User, 2nd edn, Prentice-Hall, Upper Saddle River, N.J.

Ljung, L. (2010). Perspectives on system identification, Annual Reviews in Control 34(1).

Ljung, L. (2012). System Identification Toolbox for use with MATLAB. Version 8., 8th edn, The MathWorks, Inc, Natick, MA.

Pelckmans, K., Brabanter, J. D., Suykens, J. A. \& Moor, B. D. (2005). The differogram: Non-parametric noise variance estimation and its use for model selection, Neurocomputing 69(1): 100-122.

Pelckmans, K., Suykens, J. A. \& Moor, B. D. (2006). Additive regularization trade-off: Fusion of training and validation levels in kernel methods, Machine Learning 62(3): 217-252.

Pillonetto, G. \& Bell, B. M. (2007). Bayes and empirical Bayes semi-blind deconvolution using eigenfunctions of a prior covariance, Automatica 43: $1698-1712$

Pillonetto, G., Chiuso, A. \& Nicolao, G. D. (2011). Prediction error identification of linear systems: a nonparametric Gaussian regression approach, Automatica 47(2): 291-305.

Pillonetto, G. \& Nicolao, G. D. (2010). A new kernel-based approach for linear system identification, Automatica 46(1): 81-93.

Pillonetto, G. \& Nicolao, G. D. (2011). Kernel selection in linear system identification. Part I: A Gaussian process perspective, Proc. 50th IEEE Conference on Decision and Control and European Control Conference, Orlando, Florida, pp. 4318-4325.

Quiñonero-Candela, J., Rasmussen, C. E. \& Williams, C. K. I. (2007). Approximation methods for Gaussian process regression, in D. D. L. Bottou, O. Chapelle \& J. Weston (eds), Large-Scale Kernel Machines, MIT Press, Cambridge, MA, USA, pp. 203-223.

Rao, C. R. (1973). Linear statistical inference and its applications, Wiely, Newyork.

Rasmussen, C. E. \& Williams, C. K. I. (2006). Gaussian Processes for Machine Learning, MIT Press, Cambridge, MA.

Söderström, T. \& Stoica, P. (1989). System Identification, Prentice-Hall Int., London. 